\title{
Mastectomia e o sistema de enfrentamento feminino: nuances do apoio social e familiar
}

\author{
Mastectomy and the female coping system: \\ shades of social and family support
}

\author{
Thaline Ingrid Marques Menezes Pereira' • Cícera Renata Diniz Vieira Silva ${ }^{2} \bullet$ Dayze Djanira Furtado de Galiza $^{3}$ \\ Bruno Neves da Silva ${ }^{4}$ Rayane Moreira de Alencar ${ }^{5} \bullet$ Gerlane Cristinne Bertino Véras $^{6}$
}

\begin{abstract}
RESUMO
Objetivou-se descrever as percepções de mulheres que realizaram mastectomia acerca do grupo de apoio e do suporte familiar e social. Trata-se de um estudo qualitativo de natureza descritiva, realizado com participantes de um grupo de apoio localizado em Cajazeiras/PB. Participaram do estudo 10 mulheres, entrevistadas com auxílio de um gravador de voz e de um roteiro semiestruturado. Para o tratamento dos dados optou-se pela técnica de Análise de Conteúdo. Constatou-se que o grupo de apoio foi de grande ajuda para uma melhor aceitação da doença, como também auxiliou em uma grande troca de experiências e aprendizado pelas envolvidas. No que diz respeito ao suporte familiar e social, boa parte das mulheres elencou a família como de extrema importância em todo o processo de enfrentamento. Entretanto percebeu-se que ainda há uma influência negativa direta nas relações matrimoniais. Evidencia-se a necessidade da abordagem da mastectomia de forma a refletir sobre os impactos nas dimensões das pacientes, indo de encontro, inclusive, às áreas psicológica e social.
\end{abstract}

Palavras-chave: Neoplasias da Mama; Mastectomia; Saúde da Mulher.

\begin{abstract}
:
The aim of this study was to describe the perceptions of women who underwent mastectomy on the support group and on family and social support. It is a qualitative study of descriptive nature, carried out with participants of a support group located in Cajazeiras/PB. Ten women, interviewed with a voice recorder and a semi-structured script, participated in the study. For the treatment of the data we opted for the technique of Content Analysis. It was found that the support group was of great help for a better acceptance of the disease, but also aided in a great exchange of experiences and learning by those involved. Regarding family and social support, many of the women listed the family as extremely important in the entire coping process. However, it has been realized that there is still a direct negative influence on marriage relationships. The need for a mastectomy approach is evident in order to reflect on the impacts on the patients' dimensions, even to the psychological and social areas.
\end{abstract}

Keywords: Breast Neoplasms; Mastectomy;Women’s Health.

'Enfermeira egressa da Universidade Federal de Campina Grande/Centro de Formação de Professores - UFCG/CFP. thalineingrid@hotmail.com.

${ }^{2}$ Enfermeira, mestra em saúde pública pela Universidade Estadual da Paraíba - UEPB. Docente da Universidade Federal de Campina Grande/Centro de Formação de Professores/Escola Técnica de Saúde de Cajazeiras-UFCG/CFP/ETSC.renatadiniz_enf@yahoo.com.br.

${ }^{3}$ Enfermeira, mestra em saúde coletiva pela Universidade de Fortaleza - UNIFOR. Docente da Universidade Federal de Campina Grande/Centro de Formação de Professores - UFCG/CFP. dayze_galiza@hormail.com.

${ }^{4}$ Enfermeiro egresso da Universidade Federal de Campina Grande/Centro de Formação de Professores - UFCG/CFP.nevess.bruno5@gmail.com.Autor correspondente. ${ }^{5}$ Mestranda em enfermagem pela Universidade Regional do Cariri - URCA. Docente da URCA. rayanealencar@hotmail.com.

${ }^{6}$ Enfermeira, mestra em enfermagem pela Universidade Regional o Cariri - URCA. Docente da Universidade Federal de Campina Grande/Centro de Formação de Professores/Escola Técnica de Saúde de Cajazeiras-UFCG/CFP/ETSC.gerlaneveras2@gmail.com. 


\section{INTRODUÇÃO}

A mastectomia é um procedimento cirúrgico bastante agressivo no qual ocorrerá a retirada total da glândula mamária, com o objetivo melhorar a expectativa de vida de mulheres pertencentes a populações consideradas de alto risco, sendo quase sempre inevitável em fases adiantadas do câncer de mama ${ }^{(1)}$.

Tal procedimento provoca muitas alterações na rotina de uma mulher que o realiza, gerando, inclusive, limitações na execução das atividades de vida diária e comprometimento do vínculo empregatício, devido à restrição de atividades que necessitem de força física e das dores $^{(2)}$. Ademais, tal procedimento também gera impacto na saúde psicossocial da mulher, visto a simbologia que a mama representa para o sexo feminino(3).

Contudo, continua sendo o tratamento mais eficaz para o câncer de mama, sendo normalmente associada a outros métodos terapêuticos, como a quimioterapia e a radioterapia ${ }^{(4)}$.Dessa forma, o apoio familiar e social é fundamental para a recuperação física e psíquica da mulher ${ }^{(3)}$.

Esse apoio compreende ao suporte emocional, educacional ou informativo(5), sendo de grande importância para o restabelecimento das condições de saúde, em especial por minimizar os efeitos estressores psicossociais $^{(6)}$, promover a autoestima, a valorização da pessoa humana e um melhor enfrentamento de situações difíceis relacionadas à doença ${ }^{(7)}$, o que evidencia a relevância de se discutir sobre o papel desses mecanismos de apoio na vida da mulher mastectomizada ${ }^{(4)}$.

Diante do exposto, objetivou-se descrever as percepções das mulheres que realizaram mastectomia acerca do grupo de apoio e do suporte familiar e social.

\section{MÉTODO}

Estudo exploratório e descritivo, com abordagem qualitativa, realizado no município de Cajazeiras-PB com participantes de um grupo de apoio a mulheres com histórico de câncer de mama denominado "Grupo Amigos do Peito de Cajazeiras", fundado em março de 2006 por uma enfermeira, professora universitária aposentada, que foi submetida à mastectomia.

A população do estudo foi composta por 15 mulheres que realizaram a mastectomia e participam do referido grupo de apoio. Como critérios de seleção, foram incluídas as mulheres com mais de seis meses de participação no referido grupo, entendendo-se que esse seja o período mínimo para desenvolver familiaridade com a dinâmica do mesmo, e excluídas as que não foram contatadas no período da coleta de dados, março de 2017, constituindo uma amostra com 10 mulheres.

Os dados foram coletados, após assinatura em duas vias do Termo de Consentimento Livre e Esclarecido (TCLE), mediante utilização de entrevista com auxílio de um gravador de voz e utilizando um roteiro semiestruturado contendo questões objetivas para identificar o perfil da amostra e subjetivas relacionadas à temática do estudo.

Cinco mulheres foram entrevistadas após o encontro do grupo e cinco em domicílio, com agendamento prévio e em local reservado, sem interrupções, preservando assim as informações a serem coletadas e permitindo autonomia e liberdade para responder como quisessem. A duração média das entrevistas foi de aproximadamente 20 minutos.

Após a coleta, os dados foram organizados e categorizados conforme a técnica de Análise de Conteúdo elaborada por Laurence Bardin ${ }^{(8)}$, operacionalizada mediante as seguintes etapas: pré-análise, exploração do material e tratamento dos resultados, a inferência e a interpretação. A partir desta abordagem, identificou-se uma unidade temática central a partir da qual foram construídas categorias temáticas. Todos os dados obtidos foram analisados de forma descritiva e discutidos de acordo com a literatura pertinente.

Para garantir o anonimato das participantes do estudo, atribuiu-se a estas um pseudônimo correspondente ao nome de uma flor, simbolizando a beleza das histórias e trajetórias de vida destas mulheres.

O estudo em questão é um recorte da pesquisa intitulada "Repercussão da mastectomia na vida da mulher: esferas pessoal, familiar e social" e obedeceu às normas éticas em pesquisas envolvendo seres humanos, conforme a Resolução n. ${ }^{\circ} 466 / 12$ do Conselho Nacional de Saúde (CNS), sendo o projeto de pesquisa aprovado pelo Comitê de Ética em Pesquisa da Faculdade Santa Maria (CEP/FSM) sob parecer n. ${ }^{\circ}$ I.966.598.

\section{RESULTADOS}

Participaram deste estudo um total de 10 mulheres com faixa etária entre 60 a 69 anos de idade $(30 \% ; n=3)$, com idade mínima de 40 e máxima 80 anos, média de 62 $( \pm 12,40)$, casadas $(40 \% ; n=4)$, com ensino superior completo ( $40 \%$; $n=4)$, média de 3 filhos, com renda familiar mensal de 2,7 salários mínimos (valor de referência $R \$$ 937,00), com tempo de diagnóstico de câncer e realização da mastectomia em média de 10 anos, e tempo de participação no grupo de 7 anos.

A análise das entrevistas permitiu a formulação de duas categorias temáticas a partir da unidade temática central intitulada Apoio nos contextos familiar e social da mulher mastectomizada; intituladas:A importância do grupo de apoio para o fortalecimento das mulheres e $\mathrm{A}$ mulher mastectomizada, família e sociedade.

\section{A importância do grupo de apoio para o fortalecimento das mulheres}

Esta categoria mostra como o grupo de apoio interferiu na vida das mulheres entrevistadas. 
"Bom demais! Muito bom! [...] Dá muita força, a gente conhece pessoas que tiveram a mesma doença, que passaram pela mesma situação nossa." [Margarida] "[...] O grupo é um grupo de força, de apoio, de superação. Nós passamos a força uma para a outra." [Tulipa] "É o nosso esteio de apoio para dividir nossas dores e conquistas. Trabalhar o autoconhecimento tornarmos uma pessoa melhor que valoriza mais a vida." [Narciso]

Com base nos discursos, evidencia-se a influência positiva do grupo de apoio na vida da mulher, considerando a afirmativa das mesmas que ao serem inseridas, desfrutam da possibilidade de partilhar experiências, dividir anseios e conhecer mais sobre a doença. A ideia de apoio mútuo se configura aqui como o principal aspecto relevante no processo de enfrentamento das dificuldades.

No entanto, encontram-se nas falas pontos que dificultam $\circ$ acesso de algumas mulheres a essas reuniões, o que pode levar a um enfretamento mais lento e difícil, como se pode perceber no depoimento a seguir:

"[...] Se eu pudesse estaria sempre lá, participando direto, mas eu não posso ir muito por conta das crianças e também do sol [...] $O$ horário que elas marcam é que às vezes não dá certo pra mim, até já dei opinião para mudar, porque é logo na hora que tenho que ir buscar os meus filhos [...]." [Lírio]

\section{A mulher mastectomizada, família e sociedade}

Nesta categoria destaca-se o apoio familiar e da sociedade como de grande importância para a melhoria da saúde dessas mulheres.

"Eu tive apoio tanto da família como dos amigos, como da paróquia, como de todo mundo. Então sempre fui muito bem fortalecida [...]." [Rosa]

"[...] aí a sociedade também, os amigos, todo mundo apoia, todo mundo fica solidário, aí a gente vai levando."[Girassol]

É interessante citar a relevância da presença das pessoas que convivem com a mulher acometida pelo câncer de mama, sendo imprescindível que a família perceba o quanto esse apoio é essencial para a melhoria do seu estado emocional.

No entanto, nem todas as mulheres conseguem este apoio do companheiro, sentindo-se rejeitadas, o que interfere negativamente no andamento do tratamento e aceitação da nova situação, como se pode verificar na fala abaixo.

"[...] assim, meu marido me rejeitou, ex-marido, né? A gente se separou quando eu fiz a cirurgia, ele não me acompanhava em nada, ainda me ajudava se eu pedisse, mas o casamento ficou ruim e a gente acabou." [Margarida]

No estudo em tela, observou-se também que algumas mulheres sofreram preconceito e rejeição de pessoas de convívio próximo, como pode-se verificar nas falas abaixo.
"Na família e na sociedade [...] eles em vez de ajudar, vem com preconceito, nunca acham que vai acontecer com elas." [Tulipa]

"[...] Aí então eu estranhei que elas correram quando me viram, aí depois minha prima me disse que elas achavam que câncer pega." [Orquídea]

"A sociedade você sabe como é, tem umas que é preconceituosa, outros não, [...] porque onde você mora tem muitas pessoas que se afasta, por incrivel que pareça, e dói viu?"[Cravo]

Por outro lado, observa-se que alguns aspectos positivos podem surgir, como por exemplo, a valorização da vida, fazendo com que algumas mulheres a contemplem e vivenciem com maior entusiasmo, participando de diversos tipos de grupos.

"Depois que tive o câncer passei a sair mais, porque como antes era casada, meu marido era daqueles homens de sítio que só aceita mulher dentro de casa, aí separamos e participo hoje em dia de todo tipo de grupo de idosos, digo a você que hoje sou uma mulher libertada." [Margarida]

\section{DISCUSSÃO}

Observa-se que a faixa etária das participantes revela um dos principais fatores de risco para o desenvolvimento da doença, visto que a idade acima de 50 anos é um dos mais importantes fatores de risco para o câncer de mama devido a pessoa apresentar-se mais exposta às alterações biológicas do envelhecimento ${ }^{(9)}$.

Em relação ao estado civil, apesar do Ministério da Saúde ressaltar que mulheres que não possuem companheiros parecem ser mais frágeis face às mudanças que ocorrem em sua vida cotidiana, principalmente em relação às doenças, pois elas tendem a não realizar exames de prevenção por não terem incentivo de alguém envolvido emocionalmente em sua vida, encontrou-se uma prevalência maior de mulheres casadas no estudo ${ }^{(10)}$. Este fato instiga a realização de mais pesquisas com essa população que permita esclarecer outros fatores para esse fenômeno.

Quanto ao número de filhos, a média encontrada foi inferior ao estudo de outros autores ${ }^{(2)}$, que demonstrou uma maior proteção contra a doença em mulheres que tiveram mais de 4 filhos e que amamentaram.

A maioria das mulheres entrevistadas possuía o ensino superior completo, o que está em consonância com os achados da literatura, que relatam a baixa escolaridade como fator de risco. De acordo com pesquisadores, o baixo nível de escolaridade constitui um problema para o câncer de mama, pois influencia no acesso destas muIheres a serviços de saúde, em práticas de autocuidado e na adoção de medidas de detecção precoce para a doença ${ }^{(I I)}$. Na pesquisa, a alta escolaridade foi um fator que as ajudou as mulheres a procurarem o grupo de apoio, 
para tentarem trocar experiências construtivas acerca da doença e seu tratamento.

Um grupo de apoio a mulheres mastectomizadas tem como objetivos o apoio social e o compartilhamento de informações, oferecendo um local propício ao diálogo reflexivo sobre o câncer de mama e a mastectomia, trocando experiências e dúvidas sobre o enfretamento da situação que cada uma está vivenciando, conseguindo favorecer a confiança e melhor retorno e adaptação ao convívio social( ${ }^{(12)}$. Ademais, melhora a sexualidade e a autoestima ${ }^{(7)}$, que são muito afetadas após a realização da cirurgia $^{(13)}$. $\bigcirc$ grupo de apoio promove, ainda, esperança para as mulheres ${ }^{(14)}$.

Contudo, o grande desafio de um grupo de apoio é a dificuldade da participação assídua das mulheres participantes, sendo difícil conseguir reunir todas as integrantes devido aos afazeres do dia a dia, como também foi observado em outro estudo ${ }^{(12)}$,em especial pelo horário matutino para o encontro.

Apesar das dificuldades, percebe-se que a sociedade vem criando novas formas de resolução dos problemas de saúde, buscando construir formas terapêuticas de melhorar a saúde de forma integral, minimizando o sofrimento ${ }^{(15)}$, como um grupo de apoio, que traz benefícios psicológicos, com escuta ativa e suporte profissional dos voluntários de diversas áreas assistenciais.

Pesquisadores ressaltam que a implementação de grupos em um ambiente acolhedor, no qual saberes e experiências são trocados torna-se um ambiente em que as preocupações podem ser externadas, proporcionando tranquilidade e maior disponibilidade para a aceitação do tratamento $^{(16)}$.

No Brasil, observa-se que os grupos de apoio psicossocial, assim como a família, os amigos e a religião, são sistemas que atuam de forma positiva no desempenho de novos papéis da mulher mastectomizada, emergindo como um refúgio para a defrontação da situação e superação das angústias relacionadas ao diagnóstico e tratamento do câncer de mama, sendo comprovado que a busca de auxílio é facilitada pela socialização de experiências, constituindo-se em uma maneira de conservar a autoestima elevada, acreditar na efetividade do tratamento e superar entraves relacionados com o adoecimento(6).

Nessa perspectiva, evidencia-se a importância e torna-se fundamental a criação de grupos de apoio nos serviços de saúde pública, seja na esfera municipal, estadual ou em ambas ${ }^{(17)}$.

Destarte, autores ${ }^{(3)}$ citam que a presença familiar é uma abordagem empática que precisa se atentar para as necessidades e limitações da paciente para assim, fornecer estratégias de intervenções mais eficazes para ajudá -la. $O$ amor e o afeto da família direcionam uma melhor estabilidade emocional e se constitui como um apoio na luta contra a doença, porque com isso é possível que a mulher tenha uma melhor aceitação do diagnóstico e da mastectomia.

Contudo, nem todas as mulheres conseguem este apoio, sobretudo da figura do companheiro, o que ocasiona o sentimento de rejeição ${ }^{(18)}$ e prejudica o enfrentamento da doença, que, segundo pesquisadores, possui estreita relação com a situação do convívio com o companheiro e como ela é vista por ele ${ }^{(19)}$.

Autores $^{(20)}$ relatam que acaba ocorrendo um afastamento das pessoas quando o câncer de mama é descoberto, pois elas ainda não sabem lidar com uma situação de doença ameaçadora à vida. Existem também os preconceitos ligados ao corpo da mulher mastectomizada, dificultando a autoaceitação.

O estigma social relacionado à doença e à mastectomia apresenta grande repercussão na vida da mulher, provocando aumento do sofrimento(2l).

Percebe-se a dimensão da repercussão que a mastectomia traz para a vida das mulheres, na sua autoimagem, na relação com a família e com a sociedade, iniciando com o diagnóstico, a perda da mama e, consequentemente, pelas incapacidades físicas decorrentes da retirada de músculos durante a cirurgia, problemas relacionados à sexualidade, medo da ocorrência do câncer nas filhas, sintomas da menopausa ou outros problemas do aparelho reprodutivo e a saída do emprego precocemente, afastando-a do contexto familiar e social(21-23).

Por outro lado, observa-se que alguns aspectos positivos podem surgir, como por exemplo, a valorização da vida, fazendo com que algumas mulheres a contemplem e vivenciem com maior entusiasmo, participando de diversos grupos ${ }^{(22)}$.

Frente a toda problemática vivenciada e na tentativa de preveni-la, se faz necessário a realização de ações educativas para toda sociedade no intuito de fornecer subsídio para a adesão às medidas de prevenção do câncer e suas consequências, como também favorecer o enfrentamento após o diagnóstico e tratamento, em especial da mastectomia.

Nesse sentido, destaca-se, ainda, o papel dos profissionais de saúde em contribuir com o bem-estar da mulher mastectomizada, ofertando ações pautadas na valorização de sua autoestima, medidas de prevenção, práticas de cuidado integral e possibilitando a autonomia e expressão das mesmas ${ }^{(24)}$.

\section{CONCLUSÃO}

Constatou-se que o grupo de apoio foi de grande ajuda para uma melhor aceitação da doença, como também auxiliou com uma grande troca de experiências e aprendizados por todas que participam do grupo, sendo bem visto pelas participantes do estudo. 
No que diz respeito ao suporte familiar e social, boa parte das mulheres elencaram a família como de extrema importância em todo o processo de enfrentamento, entretanto, percebeu-se que ainda há uma influência negativa direta nas relações matrimoniais. Quanto à sociedade, ainda há resquícios de um preconceito que revela a predominância do componente estético e da rejeição ao doente e a falta de conhecimento sobre a doença e o tratamento.

Este estudo apresentou algumas limitações, pois algumas entrevistas não foram realizadas pelo fato de as mulheres não estarem frequentando as reuniões, nem se encontrarem em suas residências no período da coleta de dados, justificando assim o número da amostra.

Em suma, é visível a repercussão que a mastectomia traz para a vida da mulher, por ser um tipo de cirurgia de retirada de um órgão que é a imagem de feminilidade para a mulher.Assim, evidencia-se a necessidade da abordagem da mastectomia não somente como um processo físico que culmina na cura e em uma alteração estética. É preciso refletir sobre os impactos nas dimensões das pacientes, indo ao encontro, inclusive, às áreas psicológica e social. 


\section{REFERÊNCIAS}

I. Majewskil JM, Lopes ADF, Davogliol T, Leite JCC. Qualidade de vida em mulheres submetidas à mastectomia comparada com aquelas que se submeteram à cirurgia conservadora: uma revisão de literatura. Ciênc. saúde coletiva. 2012; 17(3):707-716.

2. Lago EA, Andrade NKS, Nery IS, Avelino FVSD. Sentimentos de mulheres mastectomizadas acerca da autoimagem e alterações da vida diária. Rev. Ciência e Saúde. 2015; 8(I):15-18.

3. Nascimento KTS, Fonsêca LCT, Andrade SSC, Leite KNS, Costa TF, Oliveira SHS. Sentimentos e fontes de apoio emocional de mulheres em pré-operatório de mastectomia em um hospital-escola. Rev. Enfermagem UERJ. 2015; 23(I): 108114.

4. Bezerra KB, Silva DSM, Chein MBC, Ferreira PR, Maranhão $J K P$, Ribeiro NL, et al. Qualidade de vida de mulheres tratadas de câncer de mama em uma cidade do nordeste do Brasil. Ciênc. saúde coletiva. 2013; 18(7): 1933-1941.

5. Furlan MCR, Bernardi J,Vieira AM, Santos MCC, Marcon SS. Percepção de mulheres submetidas à mastectomia sobre o aspecto social. Cienc. Cuidado e Saúde. 2012; I I (I):66-73.

6. Ambrósio DCM, Santos MA. Apoio social à mulher mastectomizada: um estudo de revisão. Ciênc. saúde coletiva. 2015; 20(3):85I-864.

7. Otto C, Vendruscolo C, Frigo J. Mulheres mastectomizadas: relato de experiência educativa de um grupo e a sua luta por uma nova vida. Rev. Saúde Públ. 2014; 7(2):40-48.

8. Bardin L.Análise de conteúdo. São Paulo: Edições 70; 201 I.

9. Instituto Nacional de Câncer Jose Alencar Gomes da Silva. Estimativa 2016: Incidência de câncer no Brasil. 2015.

10. Ministério da Saúde (BR). Secretaria de Atenção à Saúde. Departamento de Atenção Básica. Controle dos cânceres do colo do útero e da mama. Brasília: Ministério da Saúde; 2013.

II. Ramos WSR, Sousa FS, Santos TR, Silva Júnior WR, França ISX, Figueiredo GCAL. Sentimentos vivenciados por muIheres acometidas por câncer de mama. Health Sci Inst. 20I2; 30(3):24I-248.

12. Farias LMA, Aguiar VCF, Carvalho AMF, Linhares JM, Linhares AEMS, Sousa AMM. Grupos de mulheres mastectomizadas: Construindo estratégias de cuidado. Sanare. 2015; 14(2):91-97.

13. Zhang Y, Xu H,Wang T, He J, Qiao Y,Wei J, et al. Psychosocial
Predictors and Outcomes of Delayed Breast Reconstruction in Mastectomized Women in Mainland China: An Observational Study. PLoS One. 2015; 10(12):I-I5.

14. Martins MM, Peres RS. Fatores terapêuticos em grupo de apoio a mulheres com câncer de mama. Psic. Saúde \& Doenças. 20I4; I5(2):396-408.

15. Gomes LB, Merhy EE. Compreendendo a educação popular em saúde: um estudo na literatura brasileira. Cad. Saúde Pública. 20II; 27(I):7-18.

16. Fabbro MRC, Montrone AVG, Santos S. Percepções, conhecimento e vivências de mulheres com câncer de mama. Rev Enferm UERJ. 2008; I6(4):532-537.

17. Pinheiro CPO, Silva RM, Mamede MV, Fernandes AFC. Participação em grupo de apoio: experiência de mulheres com câncer de mama. Rev. Latino-Am. Enfermagem. 2008; 16(4):733-738.

18. Rocha JFD, Cruz PKR, Vieira MA, Costa FM, Lima CA. Mastectomia: as cicatrizes na sexualidade feminina. Rev enferm UFPE on line. 2016; 10(supl 5):4255-4263.

19. Cesnik VM, Santos MA. Desconfortos físicos decorrentes dos tratamentos do câncer de mama influenciam a sexualidade da mulher mastectomizada? Rev. Esc. Enferm. USP. 20I2; 46(4): I00I-8.

20. Bandeira D, Van der Sand ICP, Cabral FB, Flores JS, Maron LC, Santos M, et al. Repercussões da mastectomia nas esferas pessoal, social e familiar para a mulher mastectomizada: uma revisão. Revista contexto e saúde. 201 I; 10(20):473-482.

21. Azevedo JJ, Bezerra KP, Morais FR, Fernandes AC, Oliveira KM, Queiroz JC. As transformações biopsicossociais em mulheres mastectomizadas. Rev enferm UFPE on line. 2016; I0(supl I):263-72.

22. Ziguer MLPS, Bortoli CFC, Prates LA. Sentimentos e expectativas de mulheres após diagnóstico de câncer de mama. Revista de Saúde Pública do Paraná. 2016; I7(I):107-I 12.

23. Ibiapina RS, Maia JM, Silva LDC, Fernandes MA, Costa Filho AAI, Fernandes RO.Aspectos psicoemocionais de mulheres pós-mastectomizadas participantes de um grupo de apoio de um hospital geral. Rev. Interdisciplinar. 2015;8(3):35-I42.

24. Oliveira AGB, Farias FF, Filho AC, Braz MR, Balbino CM, Silvino $Z R$. Detecção precoce do câncer de mama: o direito da mulher à mamografia. Revista enfermagem atual in derme. 2016; (76): 22-29. 